\title{
Ciência, Tecnologia e Inovação na Europa: uma análise do desempenho dos sistemas de inovação, com base em indicadores
}

\begin{abstract}
Maria Celeste Reis Lobo Vasconcelos
Graduação em Engenharia Química pela Universidade Federal de Minas Gerais, Mestrado em Ciências Técnicas Nucleares pela Universidade Federal de Minas Gerais, Doutorado em Ciência da Informação pela Universidade Federal de Minas Gerais e Pós doutorado no Center for Research in Innovation Management - CENTRIM, Universidade de Brighton, na Inglaterra .Professora do corpo permanente do Mestrado Profissional em Administração da Fundação Pedro Leopoldo,Minas Gerais,Brasil .

celestevasconcelos@gmail.com
\end{abstract}

\section{Editor Científico: José Edson Lara}

Organização Comitê Científico

Double Blind Review pelo SEER/OJS

Recebido em 11.11.2017

Aprovado em 22.11.2017 


\section{Resumo}

A inovação tem sido considerada vital para a competitividade das empresas e países na economia global. No caso da União Europeia - UE, é possível observar grandes esforços dos países membros para criar infraestrutura de ciência, tecnologia e inovação, tanto no nível nacional como regional. Para dar suporte a uma política voltada ao incentivo à inovação, foram criados pela UE vários relatórios de avaliação, entre eles o European Innovation Scoreboard (EIS), que apresenta uma plataforma, com indicadores que possibilitam a análise dos investimentos em capacitação, conectividade, empreendedorismo e parcerias. Este artigo tem o objetivo de analisar o desempenho dos sistemas de inovação da UE, com base em indicadores. Para isto foi realizada uma pesquisa documental descritiva. A análise revela que o desempenho em inovação da UE continua a crescer, especialmente devido aos investimentos financeiros e em recursos humanos, ao ambiente propício à inovação e aos sistemas de inovação atraentes.

Palavras-chave: sistemas de inovação; desempenho; União Europeia; C\&T\&l; indicadores.

\section{Science, Technology and Innovation in Europe: an analysis of the performance of innovation systems, based on indicators}

\section{Abstract}

Innovation has been considered vital for the competitiveness of companies and countries in the global economy. In the case of European Union - EU, it is possible to observe great efforts of the member countries to create science, technology and innovation infrastructure, at both national and regional levels. In order to support a policy aimed at encouraging innovation, a number of reports have been created by the EU, including the European Innovation Scoreboard (EIS), which presents a platform with indicators that enable the analysis of investments in training, connectivity, entrepreneurship, innovation and partnerships. This article aims to analyze the performance of EU innovation systems, based on indicators. For this a descriptive documentary research was carried out. The analysis reveals that the EU's innovation performance continues to grow, especially due to financial and human resource investments, the enabling environment for innovation and attractive innovation systems.

Key words: innovation systems; performance; European Union; S\&T\&I; indicators. 
Ciencia, Tecnología e Innovación en Europa: un análisis del rendimiento de los sistemas de innovación, basado en indicadores

\section{Resumen}

La innovación ha sido considerada vital para la competitividad de las empresas y de los países en la economía global. En el caso de la Unión Europea - UE, es posible observar grandes esfuerzos de los países miembros para crear infraestructuras de ciencia, tecnología e innovación, tanto a nivel nacional como regional. Para dar soporte a una política orientada al incentivo a la innovación, la UE ha creado varios informes, entre ellos el European Innovation Scoreboard (EIS), que presenta una plataforma, con indicadores que posibilitan el análisis de las inversiones en capacitación, conectividad, emprendimiento y asociaciones. Este artículo tiene el objetivo de analizar el desempeño de los sistemas de innovación de la UE, sobre la base de indicadores. Para ello se realizó una investigación documental descriptiva. El análisis revela que el rendimiento en innovación de la UE sigue creciendo, especialmente debido a las inversiones financieras y en los recursos humanos, al entorno propicio para la innovación y los sistemas de innovación atractivos.

Palabras clave: sistemas de innovación; rendimiento; Unión Europea; C\&T\&l; indicadores.

\section{Introdução}

Observa-se que a inovação tem sido um dos principais agentes de mudanças econômicas e sociais nos diversos países e que o sucesso das empresas tem dependido, cada vez mais, da eficácia com que incorporam os novos conhecimentos nos seus produtos e serviços. Este fato foi ressaltado no século passado por Joseph Schumpeter, cuja obra enfatiza a importância das inovações e dos avanços tecnológicos no desenvolvimento das empresas e da economia (Schumpeter, 1970).

Entretanto, o investimento em inovação tem se tornado cada vez mais alto, devido à complexidade e sofisticação de novas pesquisas. Em decorrência, vários estudos têm mostrado o grande interesse dos países e empresas na mensuração da inovação e uma grande urgência no desenvolvimento de novos indicadores que possam melhor avaliar o retorno dos investimentos em inovação.

Para dar suporte a uma política voltada ao incentivo e à mensuração da inovação, foram criados, pela União Europeia - UE, vários relatórios de avaliação como o European Innovation Scoreboard - EIS (Painel Europeu de Inovação - PEI), 
o Global Innovation Scoreboard - GIS (Painel Global de Inovação - PGI) e o Regional Innovation Scoreboard - RIS) (Painel da Inovação Regional - PAIR), que é a extensão regional do $\mathrm{PEI}$, entre outros. Estes relatórios representam hoje instrumentos para avaliação e comparação da performance inovativa dos países membros entre si e com outros países, em particular aqueles que mais investem em P\&D no mundo.

Este artigo tem o objetivo de analisar o desempenho dos sistemas de inovação da UE, com base em indicadores. Para atingir seu objetivo, o artigo foi estruturado em cinco seções, incluindo esta Introdução (seção um), que é seguida pelo referencial teórico (seção dois), que apresenta alguns conceitos de inovação, a importância de sua mensuração e o modelo de análise do relatório European Innovation Scoreboard (EIS). A seção três apresenta a metodologia utilizada na pesquisa. Já a seção quatro traz a análise e discussão dos resultados, com base nos indicadores. As considerações finais são apresentadas na seção cinco.

\section{Referencial Teórico}

Observa-se na literatura da última década um crescente interesse pelo tema inovação, como forma de se criar e manter vantagens competitivas, tanto das empresas como dos países. Conforme ressaltado por Vasconcelos e Ferreira (2009, p. 58), a inovação passou a ser prioridade na sociedade atual: "na chamada sociedade do conhecimento, o crescente fluxo de novos conhecimentos que mantêm os mercados em movimento contínuo tem requerido das empresas uma maior atenção voltada para sua capacidade inovativa".

Observa-se também uma tendência a se discutir a inovação numa perspectiva mais ampla. Várias definições têm foco na novidade e no novo: "inovação é a total exploração de novas ideias, novas para a unidade de adoção, implementadas com a intenção de fortalecer a posição competitiva de uma firma ou beneficiar seus stakeholders" (Francis, 2005, p. 44).

Sabe-se que o risco é inerente ao processo inovativo, isto é, não existe certeza de sucesso nos investimentos em inovação. Entretanto, a taxa de inovação na atividade econômica tem crescido continuamente devido ao processo de globalização e o consequente acirramento da concorrência. Outro motivo está ligado 
às exigências de desenvolvimento sustentável, às mudanças sociais e ao potencial representado pelo desenvolvimento da ciência e da tecnologia.

Bessant e Tidd (2009) ressaltam que as empresas e países buscam a inovação pois, se não mudam seus produtos e serviços, correm o risco de serem superadas pelos concorrentes, perdendo posições na competitividade global.

Alguns pesquisadores têm analisado a cultura e ambiente propício à inovação. Bruno-Faria e Fonseca (2014) realizaram uma extensa revisão de literatura sobre o tema e, depois de uma análise de 40 artigos, concluíram que este é um tema de interesse de pesquisadores em diferentes regiões do mundo, caracterizado por sua complexidade e pelo caráter sistêmico dos fatores que o determinam. Os autores concluíram que "a cultura de inovação é considerada como algo almejado nas organizações para a melhoria de seu desempenho e de sua vantagem competitiva" Concluíram ainda que o termo "é amplamente empregado, mas carece de definições apropriadas" (Bruno-Faria \& Fonseca, p. 392).

Outro aspecto que tem chamado a atenção no ambiente acadêmico e empresarial é a capacidade de inovação de empresas e países, a adequada mensuração do comportamento inovativo, bem como a mensuração de seus resultados. Como já mencionado, esta mensuração ainda se constitui em um grande desafio para as empresas e governos, devido aos altos e crescentes investimentos em inovação.

\subsection{Capacidade de inovação}

Valladares, Vasconcellos e Di Serio (2014) fizeram um levantamento bibliográfico extenso e chegaram a uma definição de capacidade de inovação com base nos seus principais fatores determinantes. Os autores validaram os fatores determinantes da capacidade de inovação apresentados na Tabela 1. 
Tabela 1

Fatores Determinantes e Resultantes da Capacidade de Inovação e seus Domínios Teóricos

\begin{tabular}{|c|c|c|}
\hline Fator & Domínio & Referências \\
\hline $\begin{array}{l}\text { Liderança } \\
\text { transformadora }\end{array}$ & $\begin{array}{l}\text { Aquela que torna seus seguidores mais conscientes da } \\
\text { importância e do valor do trabalho; ativa suas } \\
\text { necessidades de ordem superior e os induz a } \\
\text { transcender seus interesses pessoais em prol da } \\
\text { organização. }\end{array}$ & $\begin{array}{l}\text { Avolio et al. (1999); } \\
\text { Podsakoff, MacKenzie, } \\
\text { Moorman e Fetter } \\
\text { (1990). }\end{array}$ \\
\hline $\begin{array}{l}\text { Intenção } \\
\text { estratégica de } \\
\text { inovar }\end{array}$ & $\begin{array}{l}\text { Grau em que a empresa está disposta a assumir riscos } \\
\text { para favorecer a mudança, o desenvolvimento } \\
\text { tecnológico e a inovação, e a competir agressivamente } \\
\text { a fim de obter uma vantagem competitiva. }\end{array}$ & $\begin{array}{l}\text { Covin e Slevin (1989); } \\
\text { Miller (1983). }\end{array}$ \\
\hline $\begin{array}{l}\text { Gestão de } \\
\text { pessoas para } \\
\text { inovação }\end{array}$ & $\begin{array}{l}\text { Orientação da gestão de pessoas para a inovação, } \\
\text { provendo a concessão de liberdade ou autonomia de } \\
\text { atuação aos empregados, estabelecendo metas } \\
\text { desafiadoras, permitindo que decidam como alcançá-las } \\
\text { e favorecendo a autorrealização e o comprometimento } \\
\text { com os objetivos da organização. }\end{array}$ & $\begin{array}{l}\text { Amabile (1998); } \\
\text { Amabile, Schatzel, } \\
\text { Moneta e Kramer. } \\
\text { (2004); Cummings e } \\
\text { Oldham (1997); } \\
\text { Mumford et al. (2002); } \\
\text { Shalley e Gilson } \\
\text { (2004). }\end{array}$ \\
\hline $\begin{array}{l}\text { Conhecimento } \\
\text { do cliente e do } \\
\text { mercado }\end{array}$ & $\begin{array}{l}\text { Habilidade para detectar os eventos, necessidades, } \\
\text { expectativas, mudanças significativas e tendências do } \\
\text { cliente e do mercado. Perceber as mudanças do } \\
\text { mercado antes dos seus competidores fornece } \\
\text { vantagem competitiva à empresa. }\end{array}$ & $\begin{array}{l}\text { Day (1994); Kohli e } \\
\text { Jaworski (1990). }\end{array}$ \\
\hline $\begin{array}{l}\text { Gestão } \\
\text { estratégica da } \\
\text { tecnologia }\end{array}$ & $\begin{array}{l}\text { Gestão do processo de criação e desenvolvimento de } \\
\text { tecnologias, visando à criação de valor. O processo de } \\
\text { gestão tecnológica compreende cinco etapas: } \\
\text { identificação, seleção, aquisição, explotação e proteção. }\end{array}$ & $\begin{array}{l}\text { A. R. Fusfeld (2004); } \\
\text { H. I. Fusfeld (1995); } \\
\text { Phaal, Farrukh e } \\
\text { Probert (2001); } \\
\text { Gregory (1995). }\end{array}$ \\
\hline $\begin{array}{l}\text { Organicidade } \\
\text { da estrutura } \\
\text { organizacional }\end{array}$ & $\begin{array}{l}\text { Grau em que a estrutura é caracterizada pela } \\
\text { concessão de autonomia, controles flexíveis, } \\
\text { comunicação horizontal desimpedida, valorização do } \\
\text { conhecimento e da experiência e informalidade nas } \\
\text { relações pessoais. Estruturas ditas orgânicas permitem } \\
\text { resposta mais rápida às mudanças no ambiente externo } \\
\text { do que as denominadas mecanicistas. }\end{array}$ & $\begin{array}{l}\text { Burns e Stalker } \\
(1961) ; \text { Khandwalla } \\
(1977) \text {. }\end{array}$ \\
\hline $\begin{array}{l}\text { Gestão de } \\
\text { projetos }\end{array}$ & $\begin{array}{l}\text { Planejamento, provisão dos recursos, execução e } \\
\text { controle do processo de inovação. Inclui cuidadosa } \\
\text { avaliação dos projetos, análise e planejamento visando, } \\
\text { principalmente, ganhar compreensão, compromisso e } \\
\text { apoio tanto corporativo quanto do pessoal que estará } \\
\text { envolvido no projeto. }\end{array}$ & $\begin{array}{l}\text { Cooper e Kleinschmidt } \\
\text { (2007); Rothwell } \\
(1994) \text {. }\end{array}$ \\
\hline $\begin{array}{l}\text { Desempenho } \\
\text { em inovação }\end{array}$ & $\begin{array}{l}\text { Organizações inovadoras são aquelas que exibem } \\
\text { comportamento inovador consistente ao longo do } \\
\text { tempo. }\end{array}$ & $\begin{array}{l}\text { Meyer-Krahmer } \\
\text { (1984); Ritter e } \\
\text { Gemünden (2004); } \\
\text { Prajogo e Ahmed } \\
\text { (2006); Subramanian e } \\
\text { Nilakanta (1996). }\end{array}$ \\
\hline
\end{tabular}

Fonte: Valladares, P. S. D. A., Vasconcellos, M. A., \& Di Serio, L. C. (2014). Capacidade de Inovação: Revisão Sistemática da Literatura, RAC 18(5),598-626.

Estes fatores são: liderança transformadora, intenção estratégica de inovar, gestão de pessoas para inovação, conhecimento do cliente e do mercado, gestão 
estratégica da tecnologia, organicidade da estrutura organizacional e gestão de projetos. Para os autores, "organizações inovadoras são aquelas que exibem comportamento inovador consistente ao longo do tempo" (Valladares et al., p. 605).

Observa-se que, para a avaliação da capacidade de inovação, são necessárias as medições de input e output. Valladares et al (2014) ressaltam que a comparação da medição do desempenho em inovação entre concorrentes, sejam empresas ou países, é importante em estudos empíricos. A produção científica sobre indicadores e índices de inovação foi também pesquisada por Speroni, Dandolini, Souza e Gauthier (2015) que ressaltaram que o uso de indicadores permite a verificação do desempenho de organizações, regiões ou países, seja em separado ou em comparação com outras. O conhecimento sobre indicadores de inovação não é, portanto, uma tarefa trivial e diversas são as ferramentas utilizadas.

\subsection{Inovação na União Europeia}

A indústria transformadora na Europa pode ser considerada uma das forças propulsoras chaves na economia e é responsável por mais de 30 milhões de empregos, cerca de $20 \%$ do total. Este papel fundamental na economia europeia exige, de forma crucial, a modernização contínua da indústria e a inovação dos seus produtos e processos (European Commission, 2017).

Cada vez mais a inovação é vital para a competitividade das empresas e dos países na economia global. Inovação pode significar prosperidade, gera produtividade e crescimento econômico, cria oportunidades para novos e melhores empregos, possibilita a mobilidade social e é fundamental para responder aos desafios globais da sociedade. Por isto, vários países têm desenvolvido diferentes políticas e programas que deem suporte ao desenvolvimento da inovação. São crescentes os investimentos em pesquisa e desenvolvimento (P\&D) visando melhorar produtos, serviços e processos para lançamento no mercado.

Relatório recente publicado na UE estabelece uma ligação clara entre o crescimento na UE e o investimento em pesquisa e inovação, ressaltando a importância da manutenção dos esforços para aumentar o investimento no setor (Science, Research and Innovation Performance of the EU, 2016). Segundo este documento, a pesquisa e a inovação são fundamentais para a construção de um 
futuro próspero para a UE. Por conseguinte, figuram de forma proeminente na Estratégia da Europa 2020 e sustentam o progresso em relação às 10 prioridades estabelecidas para a UE, desde um novo impulso aos empregos, ao crescimento e ao investimento, ao desenvolvimento de um mercado único digital e ao desenvolvimento da União da Energia. Como já ressaltado, a UE tem muitos pontos fortes: é aberta, diversificada e possui excelentes instituições. Para 2020, a União pretende financiar a pesquisa e inovação em uma escala sem precedentes.

Os relatórios da Comissão Europeia destacam que, em tempos de profunda transformação tecnológica e social, a competitividade da economia europeia e o bem-estar dos cidadãos europeus dependem - mais do que nunca - da capacidade dos negócios se desenvolverem e soluções inovadoras serem comercializados com sucesso. A inovação aumenta a eficiência, aumenta a produtividade da empresa e proporciona grandes benefícios aos consumidores.

Como consequência, o governo europeu tem desenvolvido continuamente um grande número de iniciativas relevantes para dar suporte a esta modernização. As pesquisas realizadas procuram identificar os passos importantes e as ações necessárias para o desenvolvimento de novas tecnologias e o estabelecimento de plataformas empíricas robustas de referência, tanto de natureza qualitativa quanto quantitativa. Os resultados, que são disponibilizados em relatórios, oferecem sugestões concretas para futuras ações. Estes estudos são estratégicos e representam ferramentas importantes para os profissionais de políticas públicas (European Commission, 2016).

Um dos relatórios mais recomendados é o European Innovation Scoreboard EIS (Painel Europeu de Inovação - PEI), que fornece uma análise comparativa do desempenho da inovação nos países da UE e de outros países europeus e vizinhos regionais. Ele avalia os pontos fortes e fracos relativos aos sistemas nacionais de inovação e ajuda os países a identificar áreas em que precisam melhorar. O EIS possui uma ferramenta interativa que permite comparações personalizadas de pontuações de desempenho. É possível fazer comparações de perfis de diferentes países, bem como correlações entre os escores.

O Regional Innovation Scoreboard (RIS) - Painel da Inovação Regional - PAIR é uma extensão do EIS e avalia o desempenho da inovação das regiões europeias com base em um número limitado de indicadores. 


\subsection{European Innovation Scoreboard - EIS}

A edição de 2017 do EIS constitui um importante avanço conceitual do relatório, pois apresenta uma plataforma analítica refinada, em comparação aos relatórios anteriores. Novos indicadores foram introduzidos, possibilitando a análise dos investimentos em capacitação, conectividade, empreendedorismo e parcerias público/privado (PPP). A revisão foi fruto das interações com especialistas de renome e representantes dos Estados membros da UE.

A Figura 1 apresenta a plataforma de mensuração do EIS, usada em 2016 e revisto em 2017, com os grupos principais e dimensões.

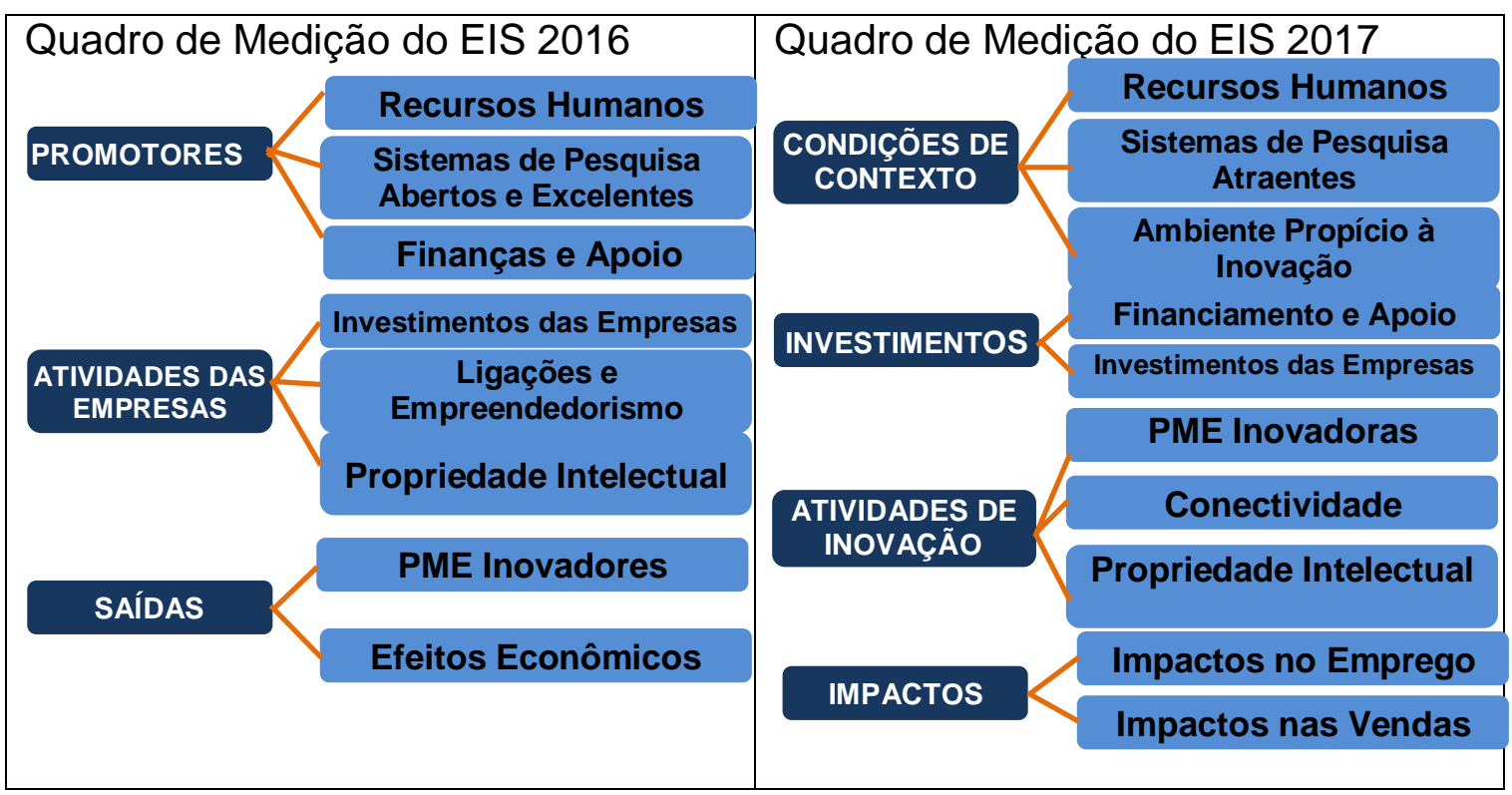

\section{Figura 1}

Quadro de Medição do EIS: Grupos Principais e Dimensões

Fonte: European Innovation Scoreboard - EIS (2017). European Commission, 2017. Recuperado em 31, outubro, de http://ec.europa.eu/growth/industry/innovation/facts-figures/scoreboards_en.

Como mostrado na Figura 1, o novo quadro de medição, utilizado em 2017, é composto por dez dimensões, incluindo uma nova dimensão relativa ao ambiente propício à inovação. A dimensão sobre os efeitos econômicos foi dividida em duas dimensões distintas, medindo o impacto da inovação no emprego e nas vendas. Ao excluir três indicadores do relatório de 2016 e incluir cinco novos, o número de indicadores aumentou de 25 para 27 este ano. Além disso, as definições foram revisadas para seis dos indicadores (European Innovation Scoreboard, 2017). 
A Tabela 2 apresenta todas as dez dimensões e os 27 indicadores do EIS. Os três indicadores constantes do relatório de 2016 eliminados estão marcados em vermelho, os nove indicadores revisados em azul e os cinco novos indicadores incluídos no relatório de 2017 em verde.

\section{Tabela 2}

Quadro de medição do EIS: indicadores

\begin{tabular}{|c|c|}
\hline $\begin{array}{c}\text { Quadro de medição EIS } 2016 \\
\text { (indicadores removidos em vermelho, } \\
\text { indicadores revisados em azul) }\end{array}$ & $\begin{array}{c}\text { Quadro de medição EIS } 2017 \\
\text { (indicadores revisados em azul, } \\
\text { novos indicadores em verde) }\end{array}$ \\
\hline 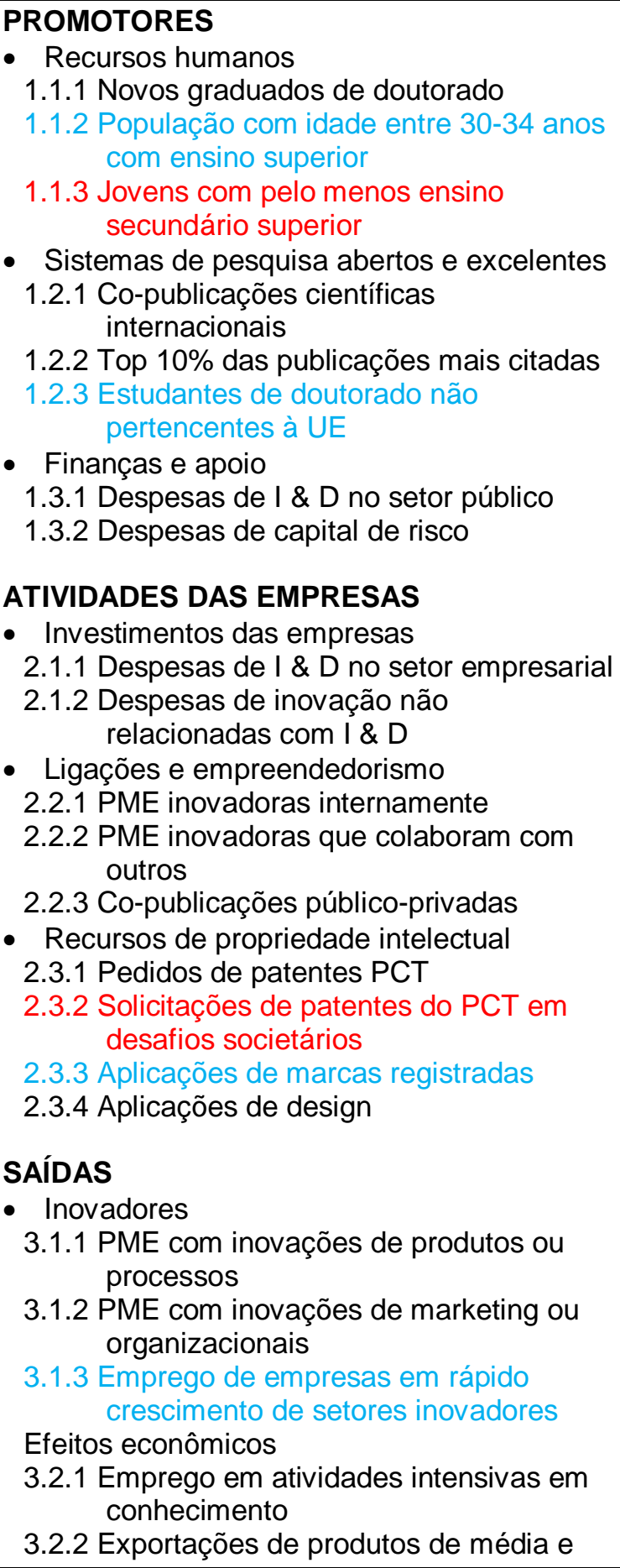 & 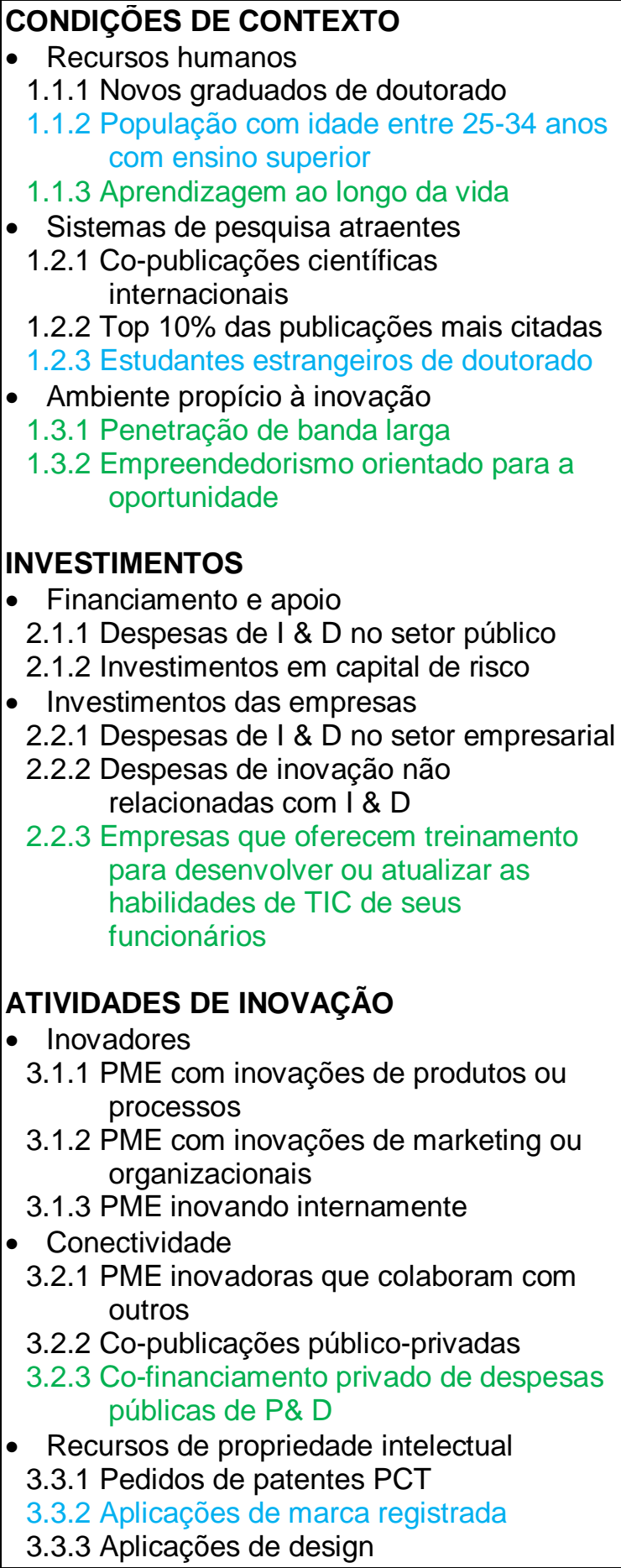 \\
\hline
\end{tabular}


alta tecnologia

3.2.3 Exportações de serviços com alto conhecimento

3.2.4 Vendas de inovações de produtos novos a mercado e de novo a empresa

3.2.5 Receitas de licenças e patentes do exterior

\section{IMPACTOS}

- Impacto no emprego

4.1.1 Emprego em atividades intensivas em conhecimento

4.1.2 Emprego empresas em rápido crescimento de setores inovadores

- Impactos nas vendas

4.2.1 Exportações de produtos de alta e média tecnologia

4.2.2 Exportações de serviços intensivos em conhecimento

4.2.3 Vendas de inovações de produtos novos a mercado e de novo a empresa

Fonte: European Innovation Scoreboard - EIS (2017). European Commission, 2017. Recuperado em 31, outubro, 2017, de http://ec.europa.eu/growth/industry/innovation/facts-figures/scoreboards_en.

O grupo "Condições de contexto" mede os principais impulsionadores do desempenho da inovação externos à empresa e abrange três dimensões da inovação: Recursos Humanos, Sistemas de Pesquisa mais Atraentes e Ambiente propício à Inovação.

O grupo "Investimentos" capta o investimento público e privado na pesquisa e na inovação e abrange duas dimensões: Financiamento e apoio e Investimento das empresas.

O grupo "Atividades de inovação" tem em conta os esforços de inovação ao nível da empresa, agrupados em três dimensões: PME Inovadoras, Conectividade e Patrimônio Intelectual.

O grupo "Impactos" mede os efeitos das atividades de inovação das empresas em duas dimensões da inovação: Impactos no Emprego e Impactos nas Vendas.

É ressaltada abaixo a relevância de alguns dos indicadores revisados ou novos, incluídos no EIS 2017;

A "Aprendizagem ao longo da vida" (1.1.3), que ressalta a importância da educação continuada e reforça a Dimensão "Recursos humanos".

O número de "Estudantes estrangeiros de doutorado" (1.2.3), que dá mais significado à Dimensão "Sistemas de pesquisa atraentes".

$\checkmark \quad$ O "Empreendedorismo orientado para a oportunidade" (1.3.2), que deixa ainda mais claro o significado da Dimensão "Ambiente propício à inovação".

$\checkmark \quad$ O número de "Empresas que oferecem treinamento para desenvolver ou atualizar as habilidades de Tecnologias da Informação e Comunicação - 
TIC de seus funcionários (2.2.3), fortalecendo o importante papel das Empresas no investimento em atividades de capacitação.

$\checkmark$ As "Exportações de serviços intensivos em conhecimento" (4.2.2), que fortalece a Dimensão relativa aos impactos das vendas.

A próxima seção apresenta a metodologia utilizada na pesquisa.

\section{Metodologia}

Este artigo tem o objetivo de analisar o desempenho dos sistemas de inovação da UE, com base em indicadores. Para isto foi realizada uma pesquisa documental descritiva, utilizando-se principalmente relatórios publicados pela União Europeia, como o EIS e o RIS, entre outros.

O EIS fornece uma avaliação comparativa do desempenho dos países da UE, e de alguns países terceiros, em matéria de pesquisa e inovação.

O RIS replica o quadro analítico refinado do EIS, na medida do possível, em termos de disponibilidade de dados. Além disso, apresenta uma análise detalhada dos grupos de desempenho e oferece uma nova ferramenta que pode ser usada para analisar e comparar diferenças entre regiões.

O modelo de análise do desempenho em inovação utilizado neste artigo foi a plataforma de mensuração do EIS, revisto em 2017 e apresentado na Figura $1 \mathrm{com}$ os quatro grupos principais e dez dimensões. Os 27 indicadores utilizados estão apresentados na Tabela 2.

\section{Análise e Discussão dos Resultados}

A análise está dividida em quatro blocos principais.

$\checkmark$ Primeiro bloco: desempenho em inovação dos países membros da UE.

$\checkmark$ Segundo bloco: comparação com competidores globais

$\checkmark$ Terceiro bloco: discussão dos resultados

$\checkmark$ Quarto bloco: tendências

\subsection{Desempenho em inovação dos países membros da EU}

A Figura 2 apresenta o desempenho dos sistemas de inovação dos países membros da UE. Os países foram classificados em quatro grupos: líderes em 
inovação (verde escuro), inovadores fortes (verde claro), inovadores moderados (amarelo) e, por último, inovadores modestos (vermelho).

As colunas nas cores verde escuro, verde claro, amarelo e vermelho mostram o desempenho dos países em 2016, utilizando os dados mais recentes para 27 indicadores. Os hifens pretos horizontais mostram o desempenho em 2015 e as colunas cinzas mostram o desempenho dos países em 2010. Foi utilizada a mesma metodologia de medição para todos os anos. As linhas tracejadas indicam os limiares entre os quatro grupos de desempenho em 2016, comparados ao desempenho dos países em 2016 com o da UE no mesmo ano.

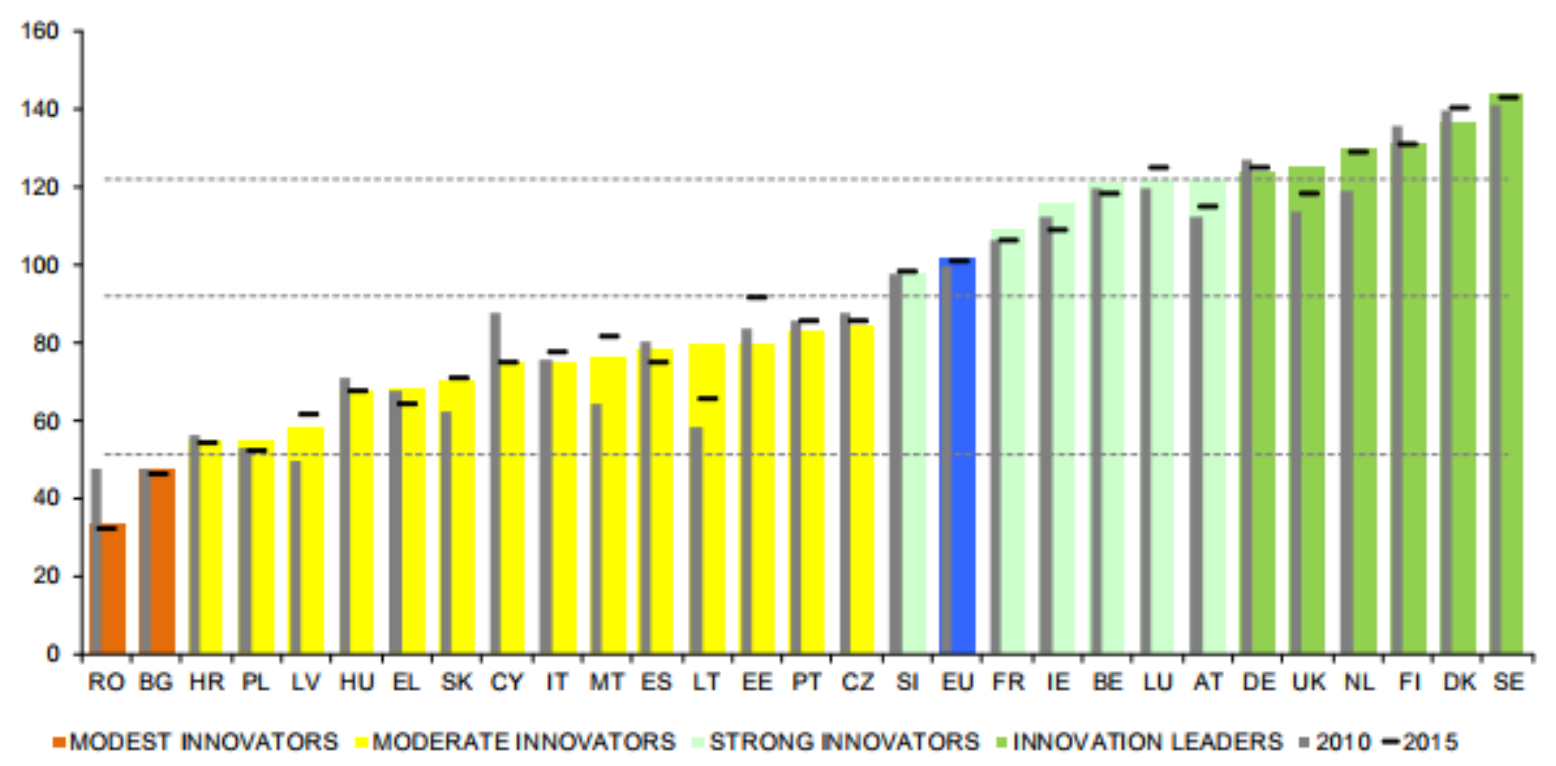

Figura 2

Desempenho dos sistemas de inovação dos países membros da UE

Fonte: European Innovation Scoreboard -EIS (2017). EN version. European Commission. Recuperado em 31, outubro, 2017 from http://ec.europa.eu/growth/industry/innovation/facts-figures/scoreboards/

Com base em suas pontuações médias de desempenho, os países foram agrupados em quatro grupos de desempenho:

$\checkmark$ Líderes em inovação com desempenho bem acima da Média da UE: Dinamarca, Finlândia, Alemanha, Holanda, Suécia e Reino Unido.

$\checkmark$ Inovadores fortes com desempenho acima ou próximo da Média da UE: Áustria, Bélgica, França, Irlanda, Luxemburgo e Eslovênia. 
$\checkmark$ Inovadores moderados, cuja média está abaixo da média da UE: Croácia, Chipre, República Tcheca, Estónia, Grécia, Hungria, Itália, Letónia, Lituânia, Malta, Polônia, Portugal, Eslováquia e Espanha.

$\checkmark$ Inovadores modestos, com desempenho bem inferior ao da média da UE: Bulgária e Romênia.

Em comparação com o relatório EIS 2010, o desempenho médio da inovação da UE aumentou no EIS 2017 em 2 pontos percentuais. Entretanto, ao se analisar cada país separadamente observa-se que alguns melhoraram o desempenho (15 países) e outros pioraram (13 países). O desempenho teve maior aumento na Lituânia, Malta, Holanda e Reino Unido e diminuiu mais no Chipre e na Romênia.

Importante também ressaltar algumas dimensões específicas de desempenho em inovação e os respectivos países líderes:

$\checkmark$ Suécia - líder em recursos humanos e na qualidade da pesquisa acadêmica;

$\checkmark$ Finlândia - líder nos indicadores relativos às finanças;

$\checkmark$ Alemanha - líder em investimentos privados em inovação;

$\checkmark$ Bélgica - líder em redes de inovação e colaboração; e

$\checkmark$ Irlanda - líder em inovação nas pequenas e médias empresas.

Os países onde a inovação registra um crescimento mais rápido são:

$\checkmark$ Letônia,

$\checkmark$ Malta,

$\checkmark$ Lituânia,

$\checkmark$ Países Baixos e

$\checkmark$ Reino Unido.

\subsection{Comparação com países vizinhos e competidores globais}

Comparando o desempenho em inovação dos países da UE com outros países europeus e vizinhos, observa-se que (EIS, 2017):

$\checkmark$ A Suíça continua sendo o país mais inovador na Europa.

$\checkmark$ A Islândia, Israel e Noruega são inovadores fortes que se apresentam acima da média da UE,

$\checkmark$ A Sérvia e a Turquia são inovadores moderados e

$\checkmark$ A Macedônia e a Ucrânia são inovadores modestos. 
A Figura 3 apresenta a comparação do desempenho dos sistemas de inovação da UE e países vizinhos usando o mesmo sistema de cores e os mesmos grupos de desempenho que a Figura 2.

O forte desempenho da Suíça está ligado ao fato de o país ter o melhor desempenho em dez dos 27 indicadores. A Suíça tem sistemas de pesquisa atraentes e tem ótimos desempenhos em todos os três indicadores da dimensão "Recursos humanos". Tem também o melhor desempenho nos indicadores "Novos graduados de doutorado", 'Aprendizagem ao longo da vida' e "PME Inovadoras".

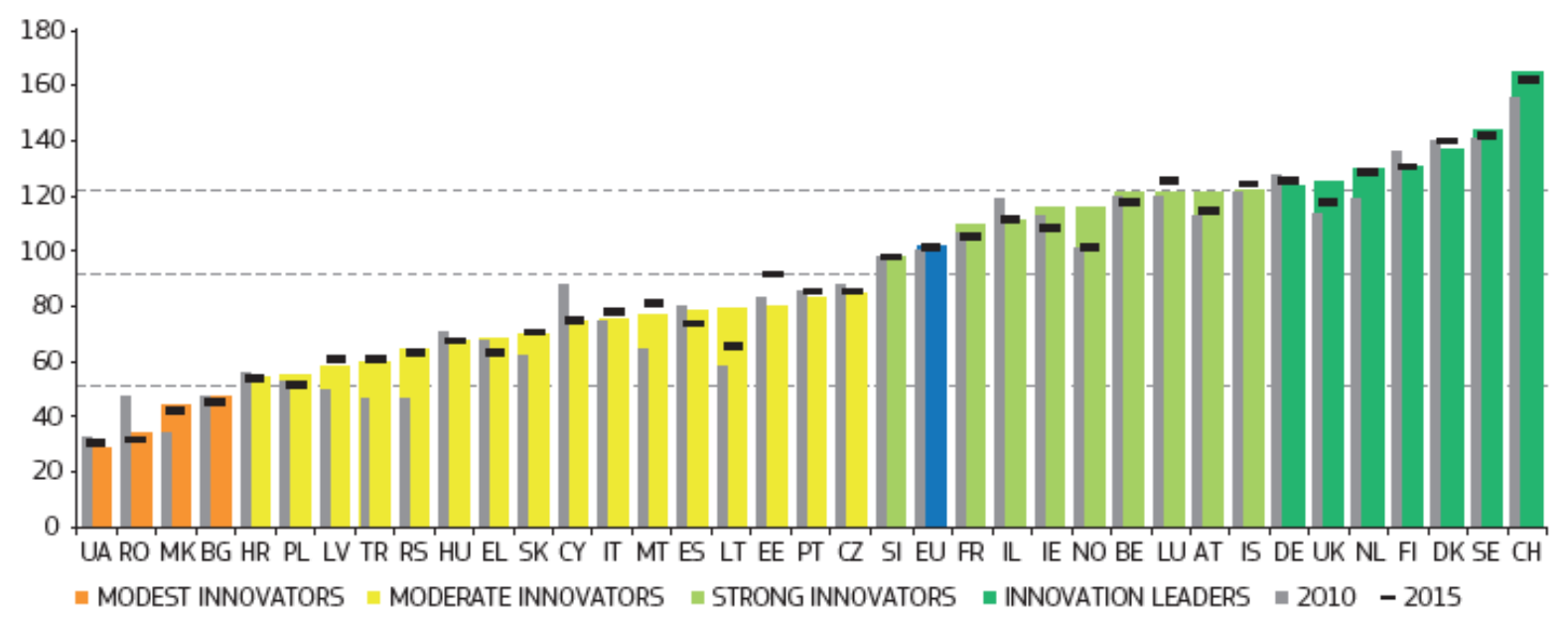

Figura 3

Desempenho dos sistemas de inovação da EU e países vizinhos

Fonte: European Innovation Scoreboard -EIS (2017). EN version. European Commission. Recuperado em 31, outubro, 2017, de http://ec.europa.eu/growth/industry/innovation/facts-figures/scoreboards/

Numa perspectiva global, o relatório fornece uma comparação da UE com alguns de seus principais concorrentes econômicos, incluindo a Austrália, os países do BRICS (Brasil, Rússia, Índia, China e África do Sul), Canadá, Japão, Coréia do Sul e os Estados Unidos (Figuras 4 e 5).

$\mathrm{Na}$ Figura 4, as barras mostram o desempenho dos países em 2016, em relação ao da UE em 2010. Na Figura 5, a variação no desempenho é medida como a diferença entre o desempenho de cada país em 2016 em relação à UE em 2010 e o respectivo desempenho em 2010 em relação à UE em 2010. 


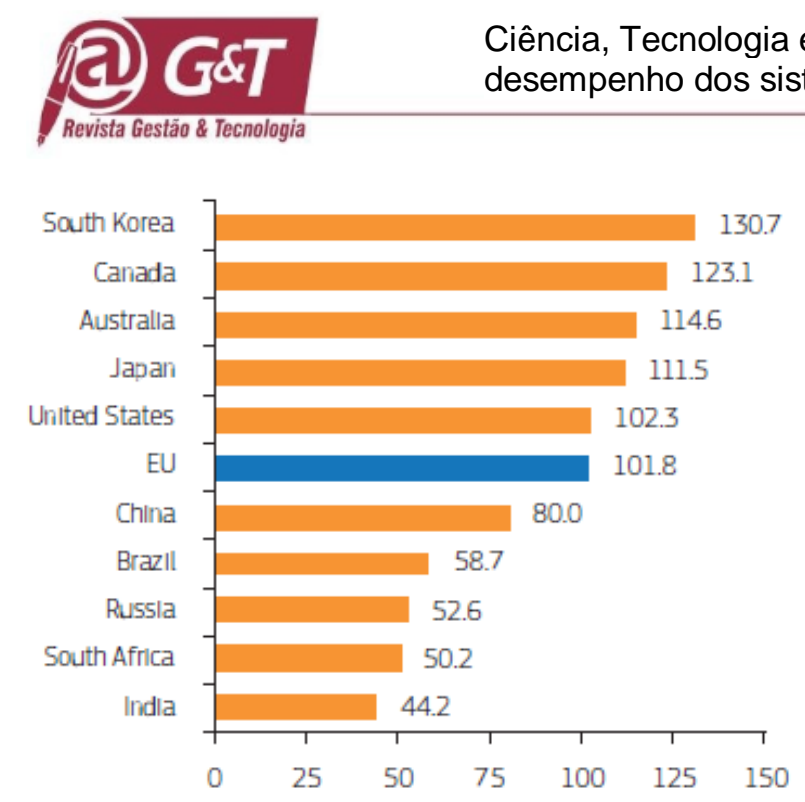

Figura 4

Desempenho Global

Fonte: European Innovation Scoreboard -EIS (2017). EN version. European Commission. Recuperado em 31, outubro, 2017 de http://ec.europa.eu/growth/industry/innovation/facts-figures/scoreboards/

Em nível global, observa-se que:

$\checkmark$ a UE é menos inovadora do que a Austrália, o Canadá, o Japão, a Coréia do Sul e os Estados Unidos;

$\checkmark$ as diferenças de desempenho da UE com o Canadá e os Estados Unidos tornaram-se menores em relação a 2010 , mas em relação ao Japão e à Coréia do Sul aumentaram;

$\checkmark$ o Japão melhorou seu desempenho mais de três vezes em relação à EU;

$\checkmark$ a Coréia do Sul melhorou seu desempenho mais de quatro vezes em relação à EU;

$\checkmark$ a UE mantém ainda uma vantagem de desempenho em relação à China, mas esta vantagem está diminuindo rapidamente;

$\checkmark$ a China melhorou mais de sete vezes em relação à EU;

$\checkmark$ O desempenho da UE é muito melhor do que o dos BRICS: Brasil, Rússia, Índia, China e África do Sul;

$\checkmark$ O Brasil é um inovador moderado e seu desempenho aumentou desde de 2010. Seu melhor desempenho está no grupo "Atividades de inovação" e no indicador "Exportações de serviços intensivos em conhecimento".

\subsection{Discussão dos resultados}

Foi ressaltado, ao longo do texto, o grande interesse dos países e empresas na mensuração da inovação, além da urgência no desenvolvimento de novos 
indicadores que possam melhor avaliar o resultado dos investimentos em inovação. Esta não é uma tarefa trivial, conforme declarado por Speroni et al (2015).

Observa-se que a UE tem mudado constantemente as dimensões que compõem o seu modelo de análise e os indicadores que compõem este modelo, na busca de resultados mais representativos. Em 2016 o modelo usado era composto de 8 dimensões. Em 2017 o modelo foi alterado para 10 dimensões. Além disto, o número de indicadores aumentou de 25 para 27 em 2017 além de ter sofrido alterações.

A Tabela 3 indica que o EIS utiliza várias dimensões similares às propostas por outros autores, por exemplo os fatores consolidados por Valladares et al (2014) para a avaliação da capacidade em inovação.

Importante ressaltar que a tendência nos modelos analisados é o aumento contínuo do número de dimensões e indicadores para avaliar o desempenho em inovação.

\section{Tabela 3}

Desempenho em Inovação

\begin{tabular}{|c|c|c|}
\hline $\begin{array}{c}\text { Fatores determinantes da } \\
\text { capacidade de inovação } \\
\text { (Valladares et al, 2014) }\end{array}$ & Dimensões do EIS (2016) & Dimensões do EIS (2017) \\
\hline 1. Liderança transformadora & 1. Recursos humanos & 1. Recursos humanos \\
\hline 2. Intenção estratégica de inovar & $\begin{array}{l}\text { 2. Sistemas de Pesquisa } \\
\text { Abertos e Excelentes }\end{array}$ & $\begin{array}{l}\text { 2. Sistemas de pesquisa } \\
\text { atraentes }\end{array}$ \\
\hline $\begin{array}{l}\text { 3. Gestão de pessoas para } \\
\text { inovação }\end{array}$ & Finanças e Apoio & $\begin{array}{l}\text { 3. Ambiente propício } \\
\text { inovação }\end{array}$ \\
\hline $\begin{array}{l}\text { 4. Conhecimento do cliente e do } \\
\text { mercado }\end{array}$ & $\begin{array}{ll}\text { 4. Investimentos } \\
\text { empresas }\end{array}$ & 4. Financiamento e apoio \\
\hline $\begin{array}{l}\text { 5. Gestão estratégica da } \\
\text { tecnologia, }\end{array}$ & $\begin{array}{ll}\text { 5. } & \text { Ligações } \\
& \text { Empreendedorismo }\end{array}$ & $\begin{array}{l}\text { 5. Investimentos } \\
\text { empresas }\end{array}$ \\
\hline $\begin{array}{l}\text { 6. Organicidade da estrutura } \\
\text { organizacional }\end{array}$ & 6. Propriedade Intelectual & 6. PME inovadoras \\
\hline \multirow[t]{4}{*}{ 7. Gestão de projetos } & PME inovadoras & 7. Conectividade \\
\hline & Efeitos Econômicos & 8. Propriedade intelectual \\
\hline & & 9. Impactos no emprego \\
\hline & & 10. Impactos nas vendas \\
\hline
\end{tabular}

Fonte: adaptado de Valladares, P. S. D. A., Vasconcellos, M. A., \& Di Serio, L. C. (2014). Capacidade de Inovação: Revisão Sistemática da Literatura, RAC, 18(5), 598-626. EIS (2016) e EIS (2017).

Outro ponto ressaltado pelo EIS (2017) é que, embora o desempenho de inovação da UE esteja melhorando, o progresso é muito lento. Muitos dos concorrentes globais estão aumentando seu desempenho em inovação a um ritmo muito mais rápido e, dentro da UE, as lacunas de desempenho permanecem 
grandes. Embora sejam observados bons progressos na educação e na pesquisa, bem como na infraestrutura de banda larga e na capacitação de TIC, os investimentos em capital de risco e o número de PMEs, que introduzem inovações, estão diminuindo fortemente.

Quanto às pequenas e médias empresas (PME), pôde-se observar que elas constituem um alvo específico das políticas de inovação na Europa. Observou-se que, quanto menor a empresa, mais ela enfrenta dificuldades para inovar ou para a comercialização de suas inovações. Como consequência, elas necessitam de políticas públicas claras de incentivo (European Commission, 2017)

Um resultado preocupante observado no EIS (2017) foi relativo à estagnação do indicador "aprendizagem ao longo da vida", que é essencial para capacitar os cidadãos num mundo em rápida mutação. O relatório concluiu que a Europa ainda não possui a inovação criadora de mercado que é necessária para transformar as melhores ideias em novos negócios e em empregos de alta qualidade. É preciso garantir, a nível local, regional, nacional e comunitário que empresas inovadoras e startups tenham acesso aos funcionários certos, com as habilidades certas, acesso à academia, a outros inovadores e parceiros de negócios. É ressaltada também a necessidade de bons investidores e de apoio político para se aventurar em novos mercados na Europa e além.

\subsection{Tendências}

O relatório de 2017 apresenta uma novidade em relação aos anos anteriores que é a discussão de tendências e mudanças esperadas. A análise explora o desempenho de tendência em 19 indicadores. Constatou-se que o aumento do desempenho é esperado para 12 desses indicadores e diminuição do desempenho para seis indicadores.

O relatório também inclui uma comparação de tendências da UE com seus principais concorrentes. No nível global, as tendências observadas nos últimos anos devem continuar, com a UE alcançando os Estados Unidos dentro de dois anos, enquanto a diferença de desempenho da UE em relação ao Japão e Coréia do Sul aumentaria, isto é, a UE deve perder terreno. Em relação à China, a tendência é de que a UE continue a perder competitividade. 
Foi destacada a iniciativa europeia de incentivo às Startups. Está sendo lançado em 2017 um Fundo Pan-Europeu de Capital de Risco, que fornecerá financiamento para projetos inovadores, de alto risco e de alto potencial. Isto será complementado por uma ação preparatória para um Conselho Europeu da Inovação, que irá promover a inovação revolucionária do mercado. Para isto é preciso ouvir, aproveitar e agregar valor às ideias dos empresários e inovadores da Europa.

Outra tendência identificada foi que, nos próximos dois anos, o desempenho da UE em matéria de inovação deverá melhorar. A maioria das empresas pensa em manter ou aumentar o nível de investimento em inovação ao longo do próximo ano. As empresas na Romênia, em Malta e na Irlanda são as que mais tendem a aumentar o seu investimento em inovação (EIS, 2017).

Foi também identificado novo tema de pesquisa em inovação na Europa, que está sendo chamado de Inovação Humanitária. Alguns pesquisadores, especialistas em inovação, estão desenvolvendo um projeto denominado "Fortalecimento do ecossistema de inovação humanitária". O primeiro relatório elaborado ressalta que a comunidade humanitária internacional está enfrentando desafios sem precedentes. O objetivo do projeto é compreender e recomendar a melhor forma de fortalecer e melhorar o ecossistema de inovação humanitária para que ele possa fazer a melhor contribuição possível para uma ajuda humanitária eficaz. Algumas prioridades deste projeto são: identificar gaps na pesquisa humanitária, desenvolver competências dos promotores de inovação, fortalecer os processos de gestão da inovação e construir uma aliança global para fortalecer o ecossistema de inovação (Ramalingam et al., 2015).

\section{Considerações Finais}

Este artigo teve como objetivo principal analisar o desempenho dos sistemas de inovação da Europa, com base em indicadores disponíveis em relatórios sobre inovação publicados pela UE. Os principais relatórios utilizados foram o European Innovation Scoreboard - EIS e o Regional Innovation Scoreboard (RIS), que é a extensão regional do EIS.

Entre as principais constatações, destacam-se: 
- a Suécia é, mais uma vez, líder da inovação na UE, seguida pela Dinamarca, Finlândia, Alemanha e Países Baixos. Estes países apresentam um desempenho bem acima da média da EU;

- a Suíça continua sendo o país mais inovador na Europa;

- o nível de inovação da UE se aproxima cada vez mais dos níveis de inovação dos EUA;

- a Letônia se tornou o país em que a inovação registra o crescimento mais rápido;

- a China apresenta os progressos mais rápidos entre os concorrentes internacionais.

O desempenho da UE, em matéria de inovação, continuou a melhorar, não obstante o fato de os progressos observados terem acontecido de modo desigual em toda a Europa. Os progressos são visíveis, mas é necessário torná-los mais homogêneos (EIS, 2017).

O EIS representa, cada vez mais, uma importante ferramenta para os formuladores de políticas, e também para os políticos, empresas e pesquisadores, visando impulsionar o apoio à inovação e, portanto, o crescimento do emprego e o crescimento da Europa.

Fica clara, nos relatórios analisados, a preocupação em se ter uma UE aberta e voltada para o exterior, que represente um destino de escolha para os investimentos globais e um centro das cadeias de valor globais. De um modo geral, observou-se que o fator determinante para um país vir a ser líder da inovação é a adoção de um sistema de inovação equilibrado, que combine um nível adequado de investimento público e privado, com parcerias eficazes entre empresas e universidades, além de uma forte base educativa e a excelência da pesquisa. É necessário que o impacto econômico da inovação se manifeste em termos de vendas e exportação de produtos inovadores, bem como na oferta de emprego.

Um ponto bastante discutido é o papel estratégico das PME. Constata-se que a Europa tem de ser um local em que as PME e as novas empresas inovadoras floresçam e prosperem no mercado único. Para tal, é necessário um esforço concentrado, no sentido de simplificar a legislação sobre os impostos, tornar as informações mais acessíveis sobre os requisitos regulamentares e trabalhar para 
criar um quadro de propriedade intelectual mais claro e favorável às PME. Os serviços inovadores e a economia criativa precisam ser incentivados.

Foi observado que continuam sendo grandes os desafios enfrentados pelos países europeus, para competir no mercado global. Uma das grandes ameaças continua sendo a China que é um país em desenvolvimento, participante dos BRICS, mas que mostra vários indicadores de inovação de país desenvolvido.

\section{Referências}

Bessant, J., \& Tidd, J. (2009). Inovação e Empreendedorismo. Porto Alegre: Bookman.

Bruno-Faria, M. de F., \& Fonseca, M. V. A. (2014). Cultura de Inovação: Conceitos e Modelos Teóricos. RAC, 18(4), 372-396.

European Commission. (2016). An analysis of drivers, barriers and readiness factors of EU companies for adopting advanced manufacturing products and technologies. Fraunhofer Institute for Systems and Innovation Research.

European Innovation Scoreboard - EIS (2016). Recuperado em 31, outubro, 2017 de http://www.knowledgetransferireland.com/About KTI/ReportsPublications/European-Innovation-Scoreboard-2016.pdf.

European Innovation Scoreboard - EIS (2017). EN version. European Commission. Recuperado em 31, outubro, 2017 de http://ec.europa.eu/growth/industry/innovation/facts-figures/scoreboards/

Francis, D. (2005). A reference model of innovation capability and implications for organizational development. Centrim, University of Brighton, UK.

Innovation (2017). European Commission. Recuperado em 08, novembro, 2017 de http://ec.europa.eu/growth/industry/innovation pt

Painel Europeu de Inovação. (2017). Recuperado em 3, novembro, 2017 de http://www.poci-compete2020.pt/noticias/detalhe/Painel Europeu Inovacao

Ramalingam, B., Rush, H., Bessant, J., Marshall, N., Gray, B., Hoffman, K., \& et al. (2015). Strengthening the Humanitarian Innovation Ecosystem. Humanitarian Innovation Ecosystem Research Project (Final Report). Centrim, University of Brighton.

Regional Innovation Scoreboard - RIS (2017). Recuperado em 8, novembro, 2017 de https://ec.europa.eu/growth/industry/innovation/facts-figures/regional en 
Schumpeter, J. (1970). Teorias econômicas de Marx a Keynes. Rio de Janeiro: Zahar.

Science, research and innovation performance of the EU: a contribution to the open innovation, open science, open to the world agenda (2016). European Commission. Recuperado em 8, novembro, 2017 de https://publications.europa.eu/pt/publication-detail/-/publication/744d5735-e1d411e5-8a50-01aa75ed71a1.

Speroni, R. M., Dandolini, G. A., Souza, J. A., \& Gauthier, F. A. O. (2015). Estado da Arte da Produção Científica sobre Indicadores e índices de Inovação. Revista de Administração e Inovação, 12(4), 49-75.

Valladares, P. S. D. A., Vasconcellos, M. A., \& Di Serio, L. C. (2014). Capacidade de Inovação: Revisão Sistemática da Literatura. RAC, 18(5), 598-626.

Vasconcelos, M. C. R. L. (2008). A Inovação no Brasil em comparação com a Comunidade Europeia: Uma Análise sobre os Desafios e Oportunidades, com base em Indicadores. XXV Simpósio de Gestão da Inovação Tecnológica, Brasília, DF., Brasil.

Vasconcelos, M. C. R. L. \& Ferreira, M. A. T. (2009). Indicadores de Inovação no Brasil e na Europa: qual é a Relação com a Gestão do Conhecimento? In C. P. A. de Paula, L. F. R. Moraes. (Orgs.). Administração contemporânea: desafios e controvérsias. (pp. 58-81). Pedro Leopoldo-MG, Fundação Cultural Dr. Pedro Leopoldo: Tavares Editora. 